\title{
Swelling of the optic nerve head: a staging scheme
}

\author{
LARS FRISÉN \\ From the Department of Ophthalmology, University of Göteborg, Sweden
}

SUMMARY A staging scheme based on ophthalmoscopic signs of disturbed axoplasmic transport is described. A study employing fundus photographs showed good reproducibility among different observers. Specificity ranged between $88 \%$ and $96 \%$, and sensitivity between $93 \%$ and $100 \%$.

The sinister implications of swelling of the optic nerve head are well known. Equally well known is a not infrequent uncertainty about the diagnosis. ${ }^{1-3}$ Congenital variants of normal anatomy and buried drusen of the optic nerve head are often difficult to differentiate from early acquired abnormality. More pronounced degrees of disc swelling are easier to recognise but correct identification of the underlying condition often presents problems. The diagnostic difficulties may in part be attributed to the lack of a detailed terminology for the various components of the ophthalmoscopic picture. A detailed scheme of description, for example in the form of a staging scheme, conceivably could facilitate evaluation. Such a scheme should also facilitate identification of a change in swelling in the individual case.

Recent experimental, ${ }^{4-7}$ and clinical and clinicopathological studies ${ }^{1289}$ have in different ways illuminated the major role of swelling of axons in the production of swelling of the optic nerve head. Swelling of axons appears to be a consequence of a local arrest of axoplasmic transport (see Hayreh ${ }^{10}$ for a review). It is not specific for raised intracranial pressure but may occur in a large variety of conditions. The observations of axonal swelling furnish a rational ground for the development of a disc swelling staging scheme. Such a scheme will be presented in the following, together with the results of a reproducibility analysis.

\section{A PROPOSED DISC SWELLING SCHEME}

The scheme is built on ophthalmoscopic signs related to an increased mass of swelling axons. It should be applicable in most conditions causing disc swelling, except those causing swelling by the occupation of the nerve head by elements normally

Address for reprint requests: Dr L Frisén, Ögonkliniken, Sahlgrenska sjukhuset, S-413 45 Göteborg, Sweden.

Received 30 May 1981, and in revised form 23 September 1981. Accepted 25 September 1981 foreign to the area (for example neoplastic cells). Any vascular components (including venous stasis, hyperaemia, haemorrhages and circumscribed infarcts) are disregarded during staging as various aetiologies and individual peculiarities allow for a large variability in expression. ${ }^{34}$

Experience attests to the great difficulty of recognising early swelling of axons within the disc itself. It is much easier to recognise a spill-over of axonal swelling at the disc border and in the adjacent peripapillary area. Therefore, the first stages depend for their definition on changes at the disc border. These include decreased translucency of nerve fibre bundles, and disruption of their normal, strictly radial arrangement. These changes lead to the formation of a subtle, grayish, faintly reticulated halo that broadens the nerve head and obscures underlying retinochoroidal detail. These changes are often best appreciated by ophthalmoscopy in red-free light. ${ }^{19}$ The more advanced stages depend for their definition on effects of anterior expansion of the swelling tissue. The intervals between successive stages have been made large enough to prevent influence by any short-term fluctuations in appearance. Stages 1 and 2 can be held to represent early disc swelling, while Stage 3 signifies moderate abnormality. Stages 4 and 5 represent severe disc swelling, and identifies a transitional stage towards progressive atrophy. Photographic examples of the various stages are given in figs 1-6. The table gives a highly condensed version of the staging scheme.

Stage 0 A normal optic disc, with blurring of the nasal and temporal disc borders by overlying nerve fibre bundles in inverse proportion to the disc diameter (that is little blurring with large discs, and vice versa). The pattern of the peripapillary nerve fibre bundles is strictly radial, without axon bundle tortuosity. Blurring of the upper and lower pole areas is disregarded because of the large normal variability. Rarely, a major vessel may be obscured 
Frisén

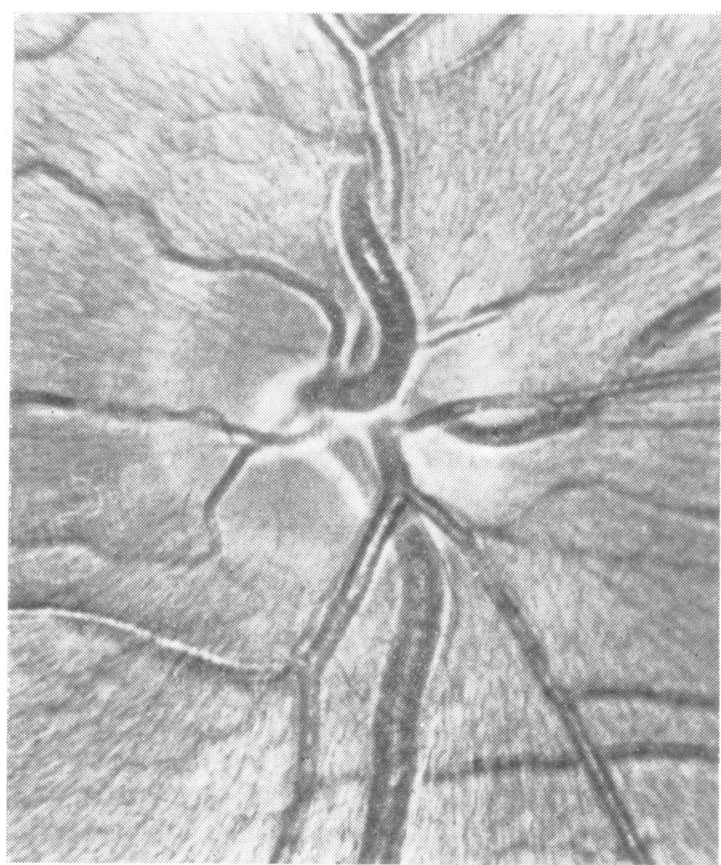

Fig. 1

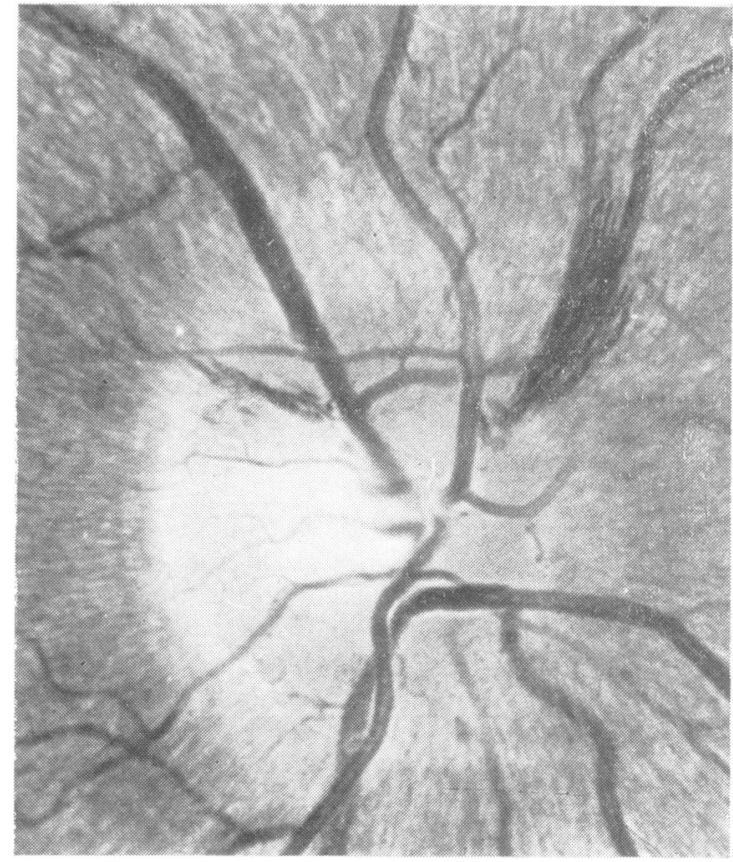

Fig. 2

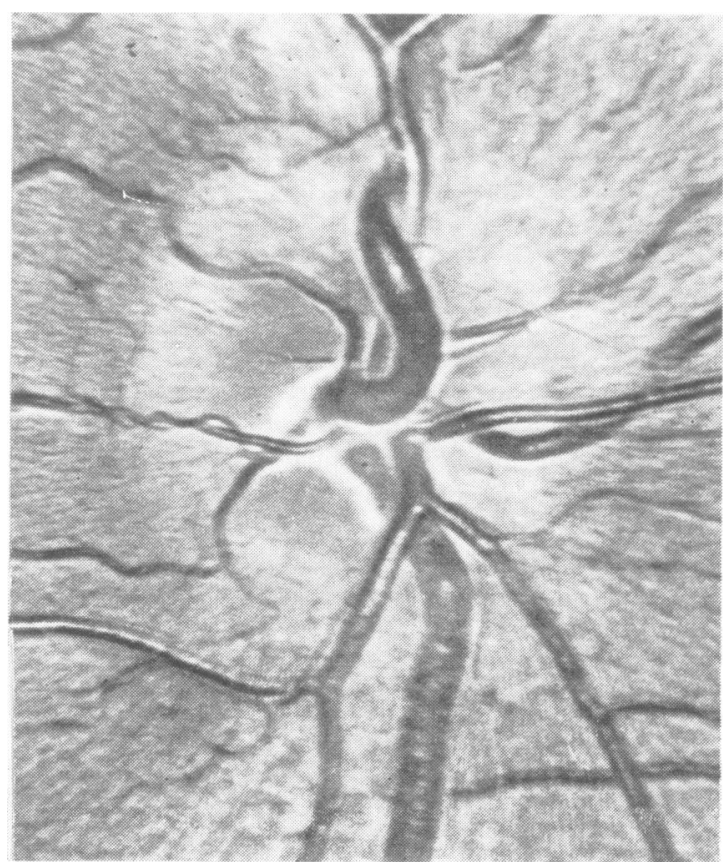

Fig. 3

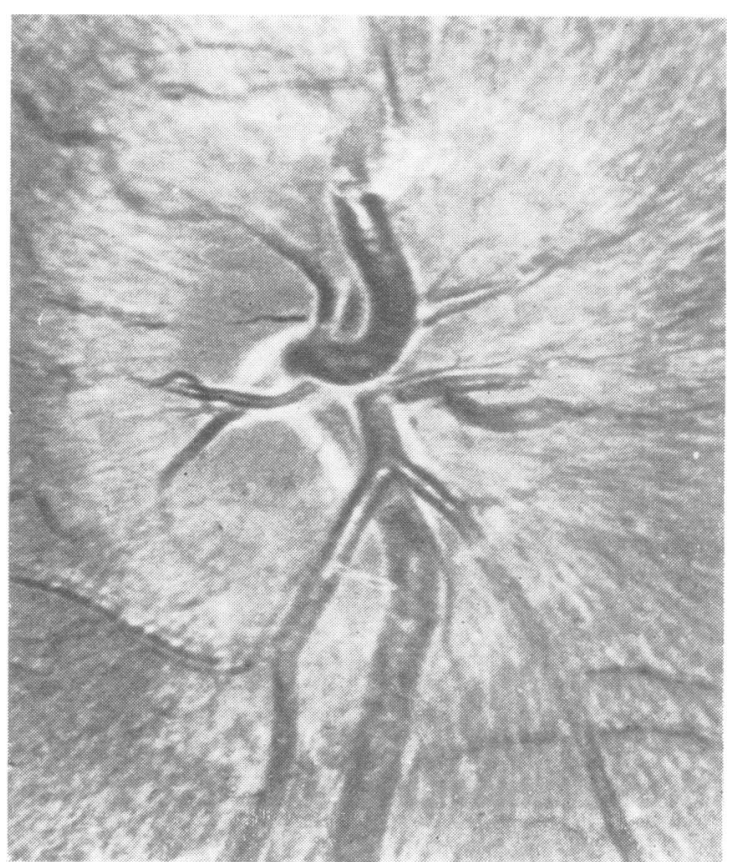

Fig. 4 


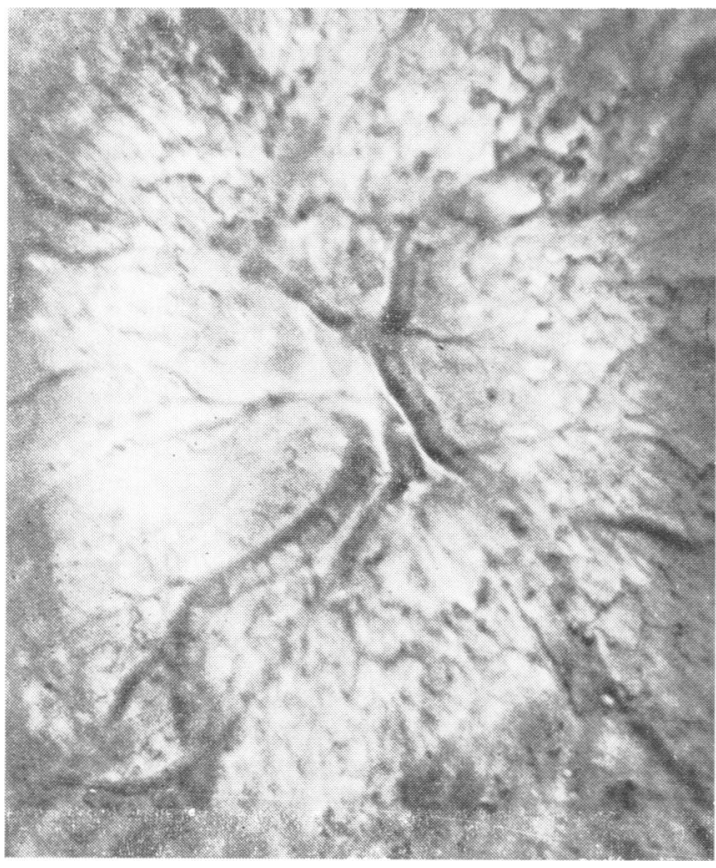

Fig. $\underline{5}$

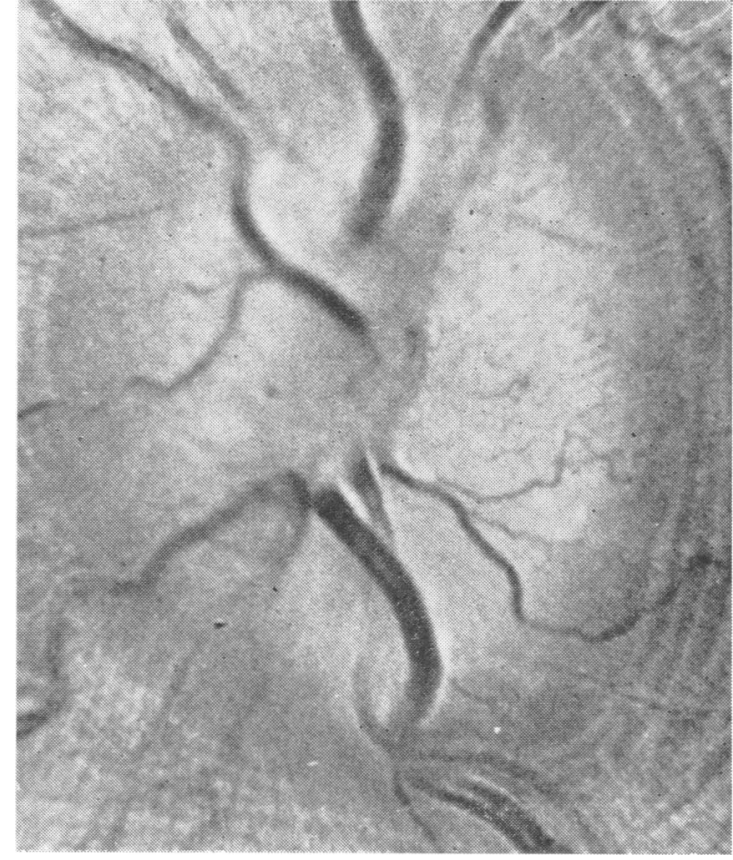

Fig.6

Figs 1-6 Fundus photographs exemplifying disc swelling stages $0-5$. Magnification $20 \times$. See text for details.

Table Stages of disc swelling

\begin{tabular}{|c|c|c|c|c|c|}
\hline \multirow[t]{2}{*}{ Stage no } & \multicolumn{5}{|l|}{ Signs } \\
\hline & nasal blur & temporal blur & peripheral TOVS* & central TOVS* or filled cup & smooth dome \\
\hline 0 & - & - & - & - & - \\
\hline 1 & - & - & - & - & - \\
\hline 2 &.-- & - & - & - & - \\
\hline 3 & - & - & + & - & - \\
\hline 4 & - & - & + & + & - \\
\hline 5 & $T$ & - & $=$ & \pm & $T$ \\
\hline
\end{tabular}

*TOVS = total obscuration of major vessel segment(s) by axons.

by overlying nerve fibres at the disc border, usually in the upper pole.

Stage 1 Excessive (in relation to the disc diameter) blurring of the nasal border of the optic disc, with disruption of the normal radial arrangement of nerve fibre bundles. The temporal margin remains normal at least in the midst of the papillomacular sector. These changes lead to the formation of a subtle grayish halo (usually best appreciated in low magnification, indirect ophthalmoscopy) along the circumference of the optic disc, with a temporal gap.

Stage 2 More pronounced changes than in Stage 1, with elevation of the nasal circumference of the nerve head, and blurring of all of the temporal margin. The halo now surrounds the disc completely. Concentric or radiating retinochoroidal folds may appear in this stage.

Stage 3 More pronounced changes than in Stage 2, with elevation also of the temporal circumference, and a clearly increased diameter of the nerve head. The elevated borders now totally obscure one or more segments of the major retinal vessels. The circumpapillary halo has an irregular outer fringe, with finger-!ike extensions.

Stage 4 More pronounced changes than in Stage 3, with elevation of the whole nerve head in 
combination with obliteration of the optic cup, or compression of the cup to a slit, or total obscuration of a segment of the central retinal artery or vein.

Stage 5 Anterior expansion of the nerve head now dominates over sideways expansion. The nerve head assumes a relatively smooth, dome-shaped protrusion, with a narrow and smoothly demarcated halo. The major retinal vessels climb steeply over the dome surface. Segments of these vessels may or may not be totally obscured by overlying swollen tissue.

The absence of notes in the staging scheme concerning hyperaemia (or pallor), haemorrhages and so-called cotton wool spots is intentional, and relates to the considerable variability of these signs between different pathogenetic conditions, and between patients with one and the same condition. In the individual case, however, it is highly appropriate to include with the stage number a note about the presence or absence of each of these signs. Clearly, the more complete the information, the easier is the recognition of both the underlying cause and any change in the appearance with time.

A given disc should be examined in turn for evidence of abnormality corresponding to Stage 1, Stage 2, Stage 3 and so on. If the signs associated with Stage 1 are absent any apparent swelling (even if pronounced) should raise the suspicion that it is due to other factors than swelling of axons (for example buried drusen). The use of more than one stage number for a given disc is discouraged. The definitions of the various stages should be stringent enough to allow confident classification with the use of one single numeral.

\section{Reproducibility test methods and results}

Three observers (one medical student, one resident in ophthalmology, and the author) staged the appearance of the optic nerve head in a series of fundus photographs, on two separate occasions. The photographs were obtained from 12 normal subjects, 12 subjects with optic disc anomalies reminiscent of acquired disc swelling, and 56 subjects with different degrees of acquired disc swelling of known aetiology. All cases had been subjected to a full neuro-ophthalmological examination by the author. Each subject contributed one fundus photograph. These were obtained on colour diapositive film, usually at $2.5 \times$ or $3.4 \times$ nominal magnification. All slides were copied in a Bowens Illumitran camera to obtain slides for projection with a uniform degree of magnification $(5 \times)$. The coded slides were mixed and projected one at a time in an ordinary slide projector, and assigned a stage number without access to clinical information. The staging procedure was repeated for each of the three examiners with an interval of at least four weeks.

All the observers commented upon the difficulty of unequivocally recognising elevation of the disc borders in some slides. This is inherent in the procedure used here: it could have been avoided only by using stereophotography. The poor depth of focus in fundus photographs also created some difficulties. It can be expected that these problems will be much reduced in clinical ophthalmoscopy, where parallactic cues and adjustment of focus can be utilised.

Two slides from patients with acquired disc swelling were deleted from the analysis because of pronounced variability in scoring between and within the observers. The variability was thought to be due to the limitations of fundus photography. For the remaining 78 slides, the reproducibility was fairly good (fig 7). In the re-test

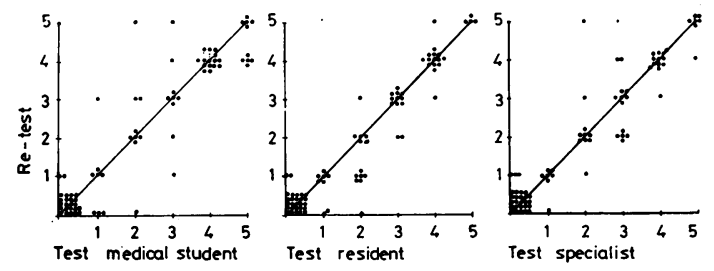

Fig 7 Reproducibility for three different observers, who staged the same fundus photographs for disc swelling on two separate occasions. Each dot represents one photograph. The diagonals represent identity of stage numbers on test and re-test.

the two senior observers gave exactly identical scores for four-fifths of the slides, and changed the score more than one unit only once. The junior observer had greater difficulties, particularly with congenitally anomalous optic discs. Nevertheless, exactly the same scores were given in three-fourths of the cases in the re-test, and a difference of one score emerged in one-fifth.

The degree of agreement between the different examiners was analysed only for the re-test data. There was exact agreement in $49 \%$ of the slides, and a difference of maximally one unit in $86 \%$. In two instances the cause of larger differences was difficulty for the junior examiner in recognising anomalous optic nerve heads; the remaining differences were fully explained by failure by one or more of the observers to adhere to the details of the staging scheme.

The most important aspect of erroneous classification pertains to falsely positive or falsely negative diagnoses of acquired optic disc swelling. There was full agreement between the observers in 22 cases about a score $=0$. An additional five slides were scored 0 by at least one observer: two of these cases had other clinical findings compatible with acquired disease. Conversely, three cases thought to be completely normal in a full neuroophthalmological examination were given score 1 by at least one observer. One of these cases had an optic disc anomaly misinterpreted by the junior observer. The other two cases were erroneously diagnosed as acquired disc swelling by failure to adhere to the details of the classification scheme. For the three observers, the specificity (defined as the fraction of normals correctly classified) ranged between 88 and $96 \%$, and the sensitivity (fraction of abnormals correctly identified) between 93 and $100 \%$. 


\section{Discussion}

There are many obstacles to the construction of a meaningful scheme for the staging of disc swelling. Major difficulties concern the lack of an established terminology for the various components of the ophthalmoscopic picture, and the very considerable variability between individuals with respect to normal morphology. There are many normal variants which create problems, particularly in the recognition of early acquired abnormality. Such variants include the "crowded disc" (the small diameter optic nerve head where nerve fibre bundles are tightly crowded at the disc border), discs with very small cup/disc ratios, discs with unusual branching patterns of major vessels, and discs with prepapillary membranes or ectopic patches of myelin. ${ }^{3}$ The most important normal variable influencing upon the evaluation of disc swelling in a given case appears to be the diameter of the scleral opening: eyes with small scleral openings tend to have considerably more blurring of the disc margins by overlying nerve fibres than do eyes with large scleral openings. ${ }^{11}$ Similar degrees of axonal swelling may therefore result in different degrees of nerve head swelling (cf figs 8-11). The evaluation and accurate staging of swelling of the optic disc

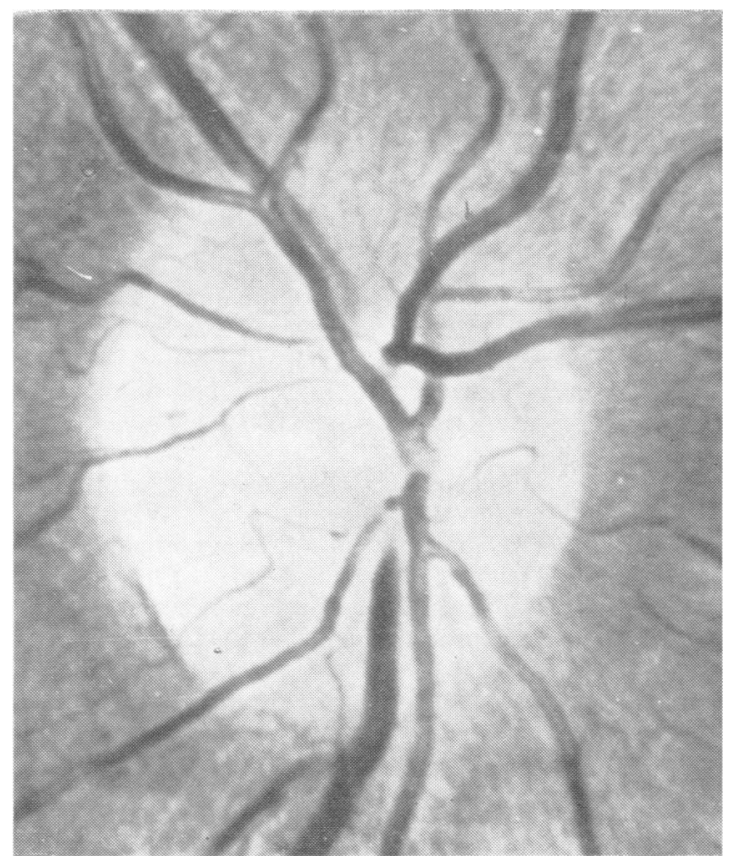

Fig. 8 obviously requires considerable attention to detail, and knowledge of normal variants which may modify the picture in the given case. High magnification ophthalmoscopy ${ }^{12}$ and refined fundus photography ${ }^{13}$ are often helpful in the evaluation of fine details. The value of fluorescein angiography may be more debatable. ${ }^{4}$ The presence or absence of buried drusen can be proved by computed tomography. ${ }^{14}$

Optic nerve heads which have lost axons through ascending or descending degeneration obviously cannot show equally pronounced swelling as normal nerve heads when axoplasmic transport is disturbed. ${ }^{4} 915$ This limitation applies not only for patients with ordinary acquired optic atrophy but also for patients with congenital optic nerve hypoplasia or drusen of the optic nerve head. ${ }^{9}$ Recognition of early disc swelling in such cases is often exceedingly difficult also for experienced examiners.

Traditional ways of assessing disc swelling by broad categorisation, ophthalmoscopic measurement of the degree of protrusion, counting the number of small vessels crossing the disc margin, and ascertaining the presence or absence of spontaneous venous pulsations, appear to have poor reliability. ${ }^{16}$ Clearly, it must be better to concentrate on direct signs of the most important element of pathology, that is axonal swelling, as done in the

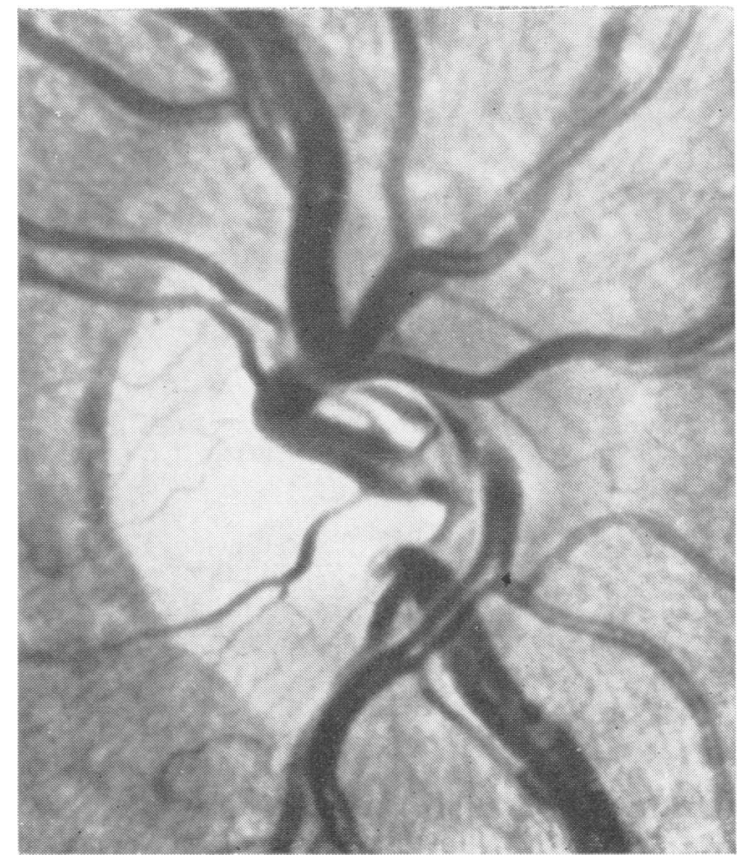

Fig. 9

Figs 8 and 9 Examples of Stage 1 disc swelling in eyes with large diameter nerve heads. $20 \times$. 


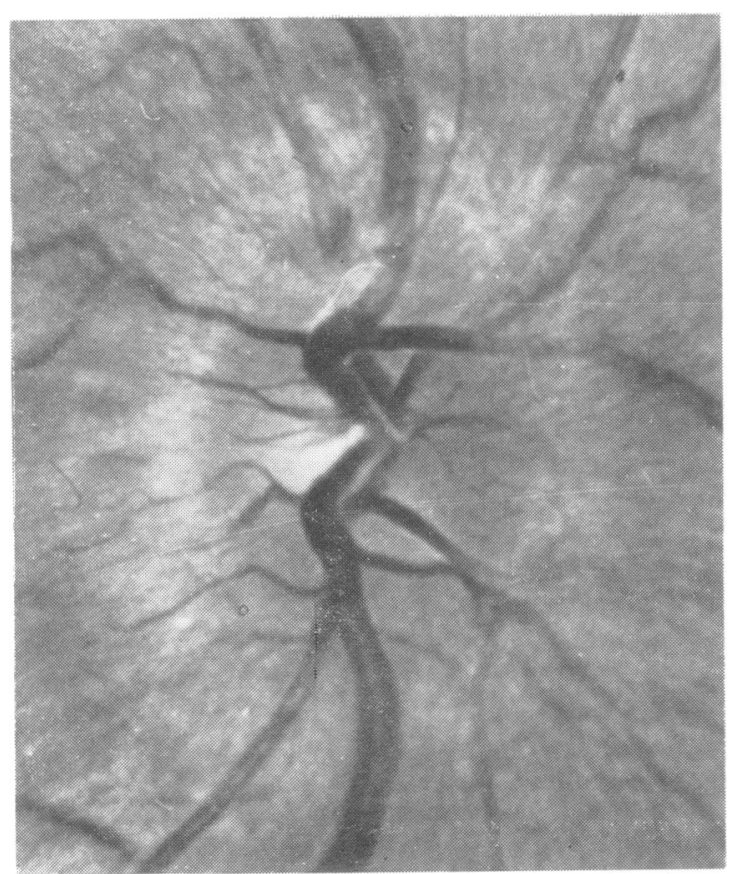

Fig. 10

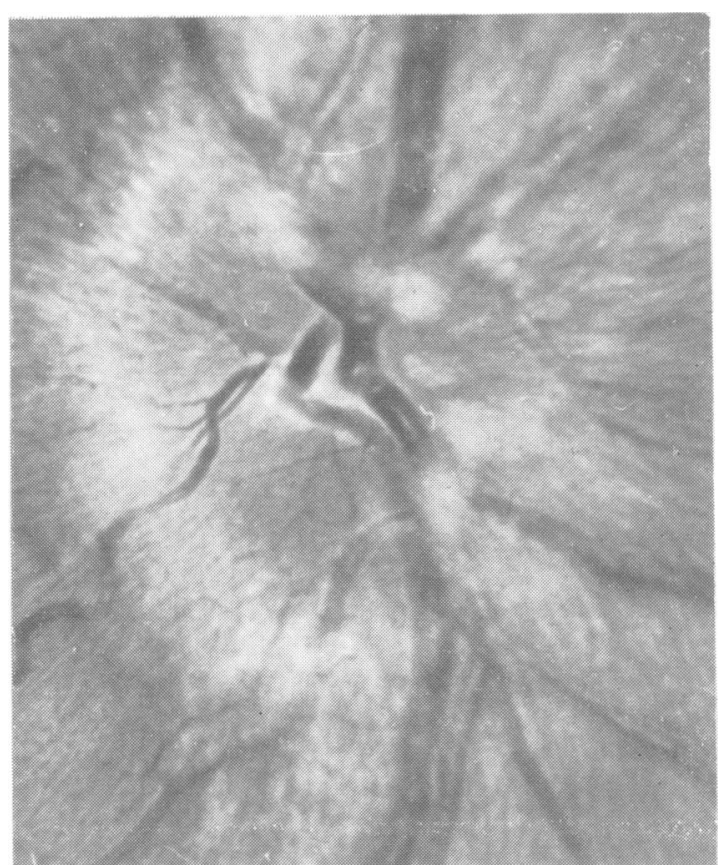

Fig. 11

Figs 10 and 11 Examples of Stage 2 disc swelling in eyes with small diameter nerve heads. $20 \times$.

proposed staging scheme. By focusing attention on fine ophthalmoscopic details the scheme should also be of aid in the differentiation of innocuous normal variants from optic nerve heads with acquired disease. On the other hand, the scheme cannot by itself improve differential diagnosis among the multitude of conditions which may cause swelling of axons. Aetiological diagnosis requires additional information. ${ }^{3}$

\section{References}

${ }^{1}$ Hoyt WF, Knight CL. Comparison of congenital disc blurring and incipient papilledema in red-free light -a photographic study. Invest Ophthalmol 1973; 12:241-7.

${ }^{2}$ Cogan DG, Kuwabara T. Papilledema. Exp Eye Res Suppl 1977;25:419-33.

3 Wirtschafter JD. Apparent enlargement of the optic disc: a pathogenetic approach to diagnosis. Perspect Ophthalmol 1977;1:274-303.

${ }^{4}$ Hayreh SS. Optic disc edema in raised intracranial pressure. V. Pathogenesis. Arch Ophthalmol 1977;95: 1553-65.

5 Wirtschafter JD, Slagel DE, Foxx WJ, Rizzo FJ. Intraocular axonal swelling produced by partial, immediately retrobulbar ligature of optic nerve. Invest Ophthalmol 1977;16:537-41.

${ }^{6}$ McLeod D, Marshall J, Kohner EM. Role of axoplasmic transport in the pathophysiology of ischae- mic disc swelling. Br J Ophthalmol 1980;64:247-61.

${ }^{7}$ Quigley HA, Addicks EM. Chronic experimental glaucoma in primates. Invest Ophthalmol Vis Sci 1980;19:137-52.

8 Tso MOM, Fine BS. Electron microscopic study of human papilledema. Am J Ophthalmol 1976;82: 424-34.

${ }^{9}$ Frisén L. Ophthalmoscopic evaluation of the retinal nerve fibre layer in neuro-ophthalmologic disease. In: Neuro-ophthalmology, Focus 1980. Smith JL, ed. New York: Masson, 1979:53-67.

${ }^{10}$ Hayreh SS. Fluids in the anterior part of the optic nerve in health and disease. Surv Ophthalmol 1978; 23:1-25.

11 Frisén L, Holmegaard L. Spectrum of optic nerve hypoplasia. Br J Ophthalmol 1978;62:7-15.

12 Frisén L. High magnification direct ophthalmoscopy. Am J Ophthalmol 1978;86:272-3.

${ }^{13}$ Frisén L. Photography of the retinal nerve fibre layer: an optimised procedure. Br J Ophthalmol 1980;64: 641-50.

${ }^{14}$ Frisén L, Schöldström G, Svendsen P. Drusen in the optic nerve head. Verification by computerized tomography. Arch Ophthalmol 1978;96:1611-4.

15 Paul TO, Hoyt WF. Funduscopic appearance of papilledema with optic tract atrophy. Arch Ophthalmol 1976;94:467-8.

${ }^{16}$ Hedges TR. Papilledema: its recognition and relation to increased intracranial pressure. Surv Ophthalmol 1975;19:201-23. 